\title{
Научные статьи
}

\section{АНАЛИТИЧЕСКОЕ ОПИСАНИЕ СЕЙСМИЧЕСКИХ СИГНАЛОВ МЕТОДОМ ФРАКТАЛЬНОГО НЕОРТОГОНАЛЬНОГО АМПЛИТУДНО-ЧАСТОТНОГО АНАЛИЗА}

\author{
(C) 2019 А.К. Рыбин ${ }^{1}$, Р.Р. Нигматуллин ${ }^{2}$, К.С. Непеина ${ }^{1}$, \\ П.А. Казначеев ${ }^{3}$, П.Н. Александров ${ }^{4}$
}

${ }^{1}$ Научная станция РАН в г. Бишкеке (НС РАН), Бишкек, 720049;

e-mail: rybin@gdirc.ru

${ }^{2}$ Казанский национальный исследовательский технический университет им. А.Н. Туполева - КАИ (КНИТУ-КАИ), Казань, 420111

${ }^{3}$ Институт физики Земли им. О.Ю. Шмидта РАН (ИФЗ РАН), Москва, 123242

${ }^{4}$ Центр Геоэлектромагнитных Исследований ИФЗ РАН (ЦГЭМИ ИФЗ РАН), Москва, Троицк; 108840

\begin{abstract}
В статье предлагается новый подход к описанию сейсмических сигналов от землетрясений на основе применения метода НАЧАСС (неортогонального амплитудно-частотного анализа сглаженных сигналов, «nonorthogonal amplitude-frequency analysis of the smoothed signals» NAFASS). Рассмотрен пример обработки записи одного из землетрясений, произошедшего на территории Тянь-Шаня, с использованием данной методики. Сигналы от этого землетрясения были зарегистрированы сейсмической станцией Карагай-Булак, входящей в состав цифровой телеметрической сети KNET, расположенной на территории Северного Тянь-Шаня. Статья представляет результаты первого этапа работы по созданию коллекции своеобразных «отпечатков» удароподобных сигналов со сложными биениями от землетрясений, которая позволит получить дополнительную информацию, характеризующую современную геодинамическую обстановку Тянь-Шаня. Подобного анализа сейсмических сигналов от землетрясений, произошедших на территории Тяньшанского региона, до настоящего времени не проводилось. Авторы надеются, что с помощью редуцированной фрактальной модели, лежащей в основе применяемого метода НАЧАСС, удастся избежать громоздких вычислений стандартными методами и непосредственно перейти к количественному описанию землетрясений в терминах общего набора параметров подгонки, создав «волновой портрет» события.
\end{abstract}

Ключевые слова: удароподобные сигналы, метод НАЧАСС, землетрясение, Тянь-Шань.

На сегодняшний день базовыми нерешенными проблемами сейсмологии являются - аналитическое описание сигналов от сейсмических событий (таких как землетрясения, взрывы, удары, микросейсмические колебания) для их дальнейшей классификации и составления прогнозов проявления сейсмичности. Благодаря заданию конкретного набора численных характеристик для представления сейсмических сигналов можно добиться уменьшения потока сохраняемой информации. В свою очередь, анализ землетрясений и их амплитудно-частотных характеристик позволяет дать представление о физическом состоянии геологической среды в момент наблюдения.

Зачастую при ручной обработке сейсмограмм возникают ошибки, влекущие за собой погрешность в определении эпицентра события (т.е. его географических координат и глубины очага). Так, например, в работе (Мухамадеева, Сычева, 2017) показано, что в результате анализа имеющейся информации для территории Киргизии по трем каталогам землетрясений (Международного сейсмологического центра 
(ISC), Института сейсмологии НАН КР (KIS) и Научной станции РАН (KNET)) выявлено наличие значительных расхождений в оценке энергетических характеристик произошедших землетрясений и определении глубины их гипоцентров. Это утверждение указывает на необходимость нового подхода к изучению записей: совершенствования методики оценки для описания землетрясений.

Численные расчеты для аналитического описания сигналов от землетрясений требуют сложного математического аппарата и вычислительных возможностей техники из-за достаточно высокой частоты дискретизации. Эта проблема до сих пор не решена. Поэтому разработка метода описания сигналов от землетрясений для возможности последующего сопоставления сейсмических событий на количественном уровне является весьма актуальной задачей, особенно для такого сейсмоактивного региона как ТяньШань (Рыбин и др., 2016; Фролова и др., 2012; Abdrakhmatov et al., 2003 и др.).

Некоторые параметры сейсмических записей, полученные на одной и той же станции, зачастую повторяются во времени, в течение недель, месяцев или даже лет. Существующая параметризация сигналов от землетрясений (набор стандартных параметров землетрясений), например, (Левин и др., 2010), очень ограничивает возможность их сравнения между собой. Поскольку источники землетрясений из одной эпицентральной зоны излучают сильно коррелированные сигналы (Geller, Mueller, 1980; Schaff, Beroza, 2004), то зачастую прибегают к распространенному в настоящее время методу кросс-корреляции волновых форм. Однако этот метод в данном случае имеет узкую область применимости (Yoon et al., 2015) в виду того, что представляющие интерес сейсмические сигналы имеют короткую продолжительность по сравнению со всей записью (обычно несколько секунд на каждом канале записи). Поэтому при корреляции приходится разбивать непрерывные данные на $\mathrm{N}$ коротких перекрывающихся окон и взаимно коррелировать все возможные пары окон. Это требует затрат значительного количества вычислительных ресурсов.

В связи с тем, что вычислительные мощности ограничены, для цифрового описания сигналов от землетрясений полезно использовать векторное пространство признаков малой размерности. Начиная с 80-х гг. XX в. для этих целей активно внедряются фрактальные методики. Фрактальные признаки присущи огромному числу природных физических процессов и структур. Модели же многих неупорядоченных процессов опираются на различные варианты случайного блуждания или динамического хаоса, также обладающих фрактальными свойствами. Фрактальные модели не всегда поддаются аналитическому исследованию, но могут быть построены по простым правилам с возможностью несложной компьютерной реализации (Зосимов, Лямшев, 1995). Отметим, что такой подход значительно отличается от традиционных методов рутинной обработки сейсмограмм. Стандартные методы основаны на спектральном подходе, то есть так или иначе они основаны на разложении сигнала по гармоническим составляющим. Как утверждает Любушин А.А. (2007, с. 8): «... учет нестационарности спектральными методами возможен только путем оценок в скользящем временном окне. Однако при этом всплески усредняются, а моменты общих всплесков могут быть определены только с точностью, равной длине временного окна. В то же время слишком сильное уменьшение длины временного окна делает спектральные оценки неустойчивыми и повышает влияние чисто шумовых компонент».

Предлагаемый метод неортогонального амплитудно-частотного анализа сглаженных сигналов (НАЧАСС, «nonorthogonal amplitudefrequency analysis of the smoothed signals», NAFASS) представляет собой метод подбора аналитической/подгоночной функции, основанный на свойстве фрактальности, поможет лучше понять внутреннюю структуру выбранного сигнала от землетрясения и извлечь дополнительную информацию о его низкочастотной огибающей, что может использоваться как специфический «отпечаток» (fingerprint), описывающий конкретное событие. Вместо прямого сравнения осциллограмм сначала выполняем функцию извлечения для каждой формы волны в компактный «отпечаток», который сохраняет только свои ключевые отличительные признаки. «Отпечаток» служит «ключом» для волновой формы; таким образом, две аналогичные волновые формы должны иметь одинаковые «отпечатки», и два разнородных сигнала должны иметь разные «отпечатки». Интерес к использованию метода НАЧАСС также обусловлен тем, что исследования такого рода для сигналов от землетрясений до сих пор не проводились, в том числе и для территории Центрального и Северного Тянь-Шаня.

\section{МЕТОДЫ ИССЛЕДОВАНИЯ. ОПИСАНИЕ АЛГОРИТМА}

Численное описание сигнала удобно осуществить, как говорилось выше, с помощью оценки фрактальной размерности. Сейсмический сигнал от землетрясения является удароподобным сигналом (УПС, англ. «blow-like signals», BLS) со сложным биением (Nigmatullin et al., 2015). Под УПС понимается такой сигнал, который 


\section{АНАЛИТИЧЕСКОЕ ОПИСАНИЕ}

содержит высокочастотную (ВЧ составляющую), расположенную между двумя низкочастотными (НЧ) огибающими (рис. 1).

Очевидно, что свойства сглаженного сигнала зависят от масштаба сглаживания. Поэтому так важно правильно выбрать размер скользящего временного окна, в зависимости от которого результаты сглаживания получаются более или менее изменчивыми. Часто используют вейвлет-преобразования, поскольку они дают возможность рассматривать свойства сигнала как в частотной, так и во временной областях. Применениям непрерывного и дискретного вейвлет-преобразований для прогнозирования временных рядов и для обработки сигналов посвящены многочисленные публикации (Короновский, Храмов, 2003; Любушин, 2007; Малла, 2005; Новиков и др., 2005; Родионов, 2016; Федер, 1991 и др.). Однако в данном исследовании основной акцентируется внимание на получении функции для описания общей формы сигнала, а не его локальных особенностей. В таком случае фрактальный подход более уместен.

При фрактальном подходе исключается процедура подбора длины окна «скользящего среднего» и его формы (прямоугольное окно, вейвлетпреобразование, окно Хэмминга, окно Кайзера (Новиков и др., 2005) и другие функциональные окна). Однако возникает необходимость выбора универсальной фрактальной величины, по которой будет происходить описание УПС. Большинство УПС по своей природе имеют фрактальное происхождение. Это означает, что при разумном сжатии или масштабировании этих сигналов они сохраняют примерно ту же исходную структуру. Это свойство масштабирования может быть про- верено на многих типах различных сигналов. Для описания этих сигналов есть возможность разделить весь сигнал с помощью модифицированного Фурье-разложения на три составляющих: две из которых представляют собой огибающие (нижнюю и верхнюю), между которыми «зажат» сигнал, и одна - высокочастотные (ВЧ) биения. После чего осуществляется поиск амплитудночастотной характеристики (АЧХ) для этих двух огибающих на основе метода НАЧАСС, который был предложен в работе (Nigmatullin, 2014). Сравнение огибающих (например, преобразование Гильберта) для сейсмических сигналов успешно применялось в работах Асминга В.Э. (2004), Баранова С.В. (2002), Конечной Я.В. (2015), Морозова А.Н. (2008), Морозова Ю.В. и Спектра А.А. (2017) и др. Предлагаемый алгоритм имеет более общий характер, и может применяться к широкому классу УПС. Метод был успешно применен для описания биофизических и экономических данных, имеющих также многочастотную структуру. Для дальнейшего удобства разделим описание предложенного алгоритма на несколько основных шагов.

Для демонстрации эффективности применения метода НАЧАСС была взята запись (от 10 ноября 2017 г.) широкополосного трехкомпонентного сейсмометра STS-2 с частотой оцифровки 100 Гц с пункта Карагай-Булак (сокр. КВК) сейсмологической сети KNET (http://www.gdirc. $\mathrm{ru} / \mathrm{ki} /$ napravlenija-issledovanij/sejsmologicheskienabljudenija), функционирование которой обеспечивает Научная станция РАН в г. Бишкеке (Березина и др., 2013). Стоит отметить, оно относится к рою землетрясений из определен-

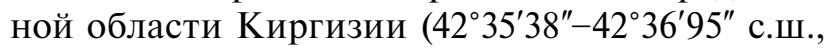

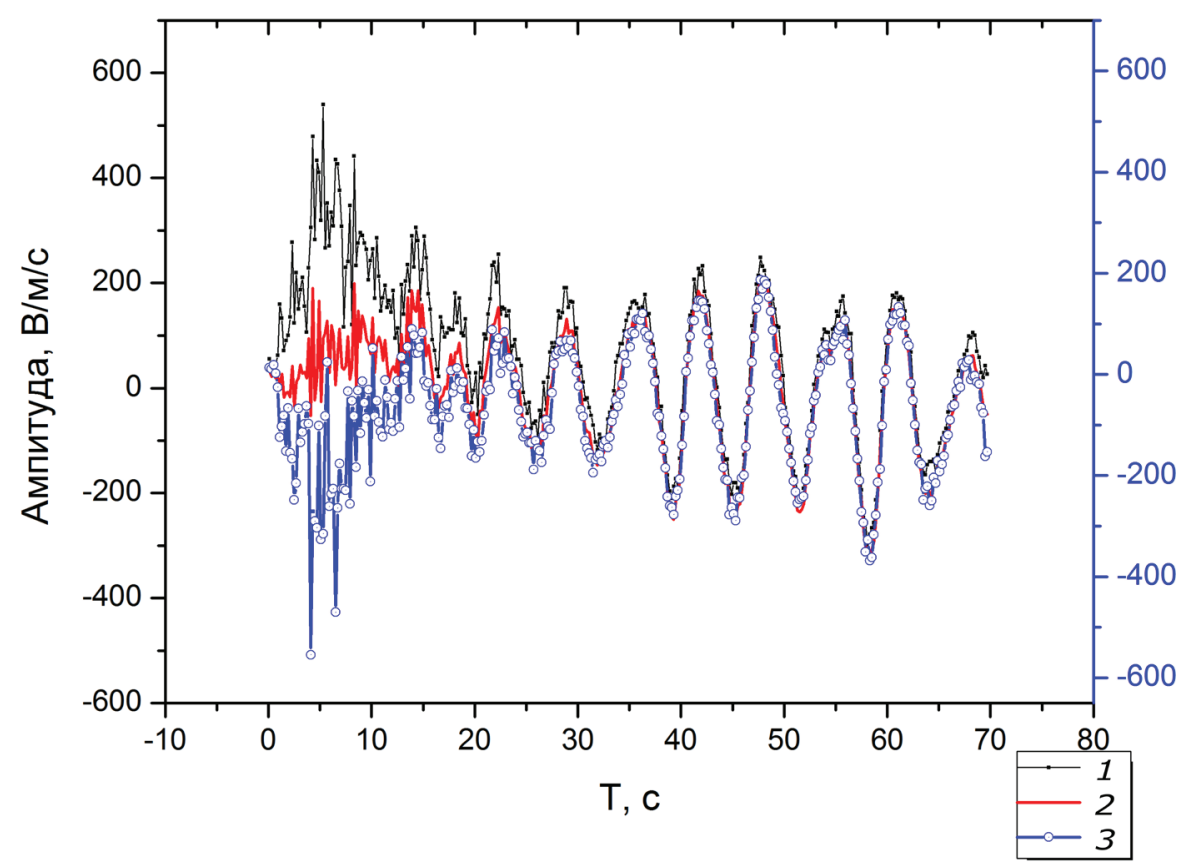

Рис. 1. Типичный сигнал, представляющий запись удароподобного сигнала (УПС) со сложным биением для случайного землетрясения после процедуры редукции в $\mathrm{M}=20$ раз показаны линиями: огибающие сигнала землетрясения 1 - верхняя, 3- нижняя, 2 - биения, редукция высокочастотной (ВЧ) составляющей.

Fig. 1. A typical signal representing a blow-like signal (BLS) with a complex beating for a random earthquake after the reduction procedure where $M=20$ is represented as the lines: earthquake signal envelopes 1 - upper, 3 - lower, 2 - beatings, reduction of the high-frequency (HF) component. 


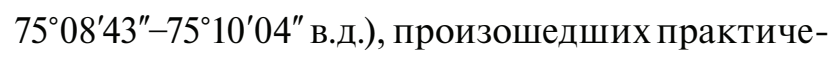
ски друг за другом (в один и тот же день). Выбранное землетрясение было первым и наиболее крупным среди роя других землетрясений, зарегистрированных из одной эпицентральной зоны. Рассматриваемое землетрясение характеризуется следующими параметрами: время в очаге $\mathrm{T}_{0}=00: 41: 52.43$ (UTC) 21.04.2017, координаты $\varphi=42^{\circ} 35^{\prime} 22.90^{\prime \prime}$ с.ш., $\lambda=75^{\circ} 10^{\prime} 02.30^{\prime \prime}$ в.д., глубина $\mathrm{H}=13.51$ км, энергетический класс $\mathrm{K}=11.55$, магнитуда $\mathrm{M}=4.2$. В данном исследовании заведомо взяты «вырезанные» из непрерывной записи землетрясения ограниченной длины (1 минута).

\section{МЕТОДИКА}

Эта процедура была подробно описана в предыдущих работах (Nigmatullin, 2014, 2015). Согласно этой процедуре, сначала следует представить сигнал в виде отрезков приблизительно равной длины. Каждый отрезок сократить до характерных точек, которые останутся инвариантными относительно перестановок среди оставшихся точек. Затем переходят к процедуре оптимального сглаживания. Следующим шагом является подбор подгоночной функции, описывающей редуцированный сигнал. В данном случае в качестве подгоночной функции используется НАЧАСС разложение, представляющее собой совокупность всевозможных обобщенных биений. Под основными составляющими этих биений подразумеваются мода $k$, низкочастотная и высокочастотная огибающие. Именно подбор биений для описания НЧ и ВЧ огибающих является трудоемкой задачей.

Шаг 1. Редукция к трем инвариантным точкам. Для доказательства свойства фрактальности представленных дискретных сигналов необходимо представить сигнал в виде редуцированного сигнала. К основным фрактальным свойствам относятся иерархичность и подобие. Таким образом, остается только доказать, что разбиение на отрезки и последующая процедура редукции не искажают сигнал, а являются вложенной копией, а значит, сигналы фрактальны. Для этого выбирается группа $b$-точек $\left(b_{1}, b_{2}, \ldots, b_{M}\right)$, где $M<<N$ (где $N$ равно начальному числу точек данных). Численное тестирование на примере функции Вейерштрасса-Мандельброта (Федер, $1991)$ показало, что величину $b$ можно взять из интервала $M \in[10,60]$, принимая во внимание, что исходная величина $N$ примерно на два порядка превосходит эту величину и задается в интервале [6000, 7000]. Поэтому разделим весь сигнал на сегменты (группы $b$-точек), имеющие в среднем 30-40 точек. Затем для каждого выбранного набора вычислялись 3 инвариант- ные точки: $b_{\text {max }}, b_{\text {mean }}, b_{\text {min }}$, которые инвариантны относительно всевозможных перестановок всех точек, образующих данный набор, где:

$b_{\max }=\max \left(b_{1}, b_{2}, \ldots, b_{M}\right)-$ максимальные значения для группы $b$-точек;

$b_{\text {mean }}=$ mean $\left(b_{1}, b_{2}, \ldots, b_{M}\right)-$ средние значения для выбранной группы;

$b_{\min }=\min \left(b_{1}, b_{2}, \ldots, b_{M}\right)-$ минимальные значение для группы $b$-точек.

Эти точки остаются инвариантными относительно перестановок среди оставшихся точек, составляющих выделенный сегмент $(b=30-40)$. Процедура редукции к трем инвариантным точкам позволяет отделить верхнюю и нижнюю огибающие сигнала землетрясения, сгладить их и далее представить составляющие редуцированного сигнала в форме НАЧАСС разложения. Процедура редукции помогает перейти к другому набору «жирных» точек $r=0,1, \ldots, R-1$, где $R=[N / M]$ ([...] соответствует выделению целочисленной части), которые уменьшают начальное число данных $N$ и сохраняют исходную форму выбранного сигнала. Обычно для сохранения желаемой формы выбранного сигнала количество выбранных точек $M$ должно удовлетворять условию $M \in(3, N / 100)$. Эта процедура показана на рис. $2(N=7000, M=20, R=350)$.

Шаг 2. Сглаживание и подгоночная функция. Процедура оптимального линейного сглаживания (ПОЛС, procedure optimal linear smoothing, POLS), используемая для целей сглаживания, была описана в работах (Nigmatullin, 2014, 2018). Вначале используется выражение:

$$
\begin{aligned}
& Y_{j}=Y_{j}(N, w)=\frac{\sum_{i=1}^{N} K\left(\frac{x_{j}-x_{i}}{w}\right) y_{i}}{\sum_{i=1}^{N} K\left(\frac{x_{j}-x_{i}}{w}\right)_{i}}, K(t)=\exp \left(-t^{2}\right), \\
& Y_{j}(N, w)=\left\{\begin{array}{l}
y_{j}, w \rightarrow 0 \\
\frac{1}{N} \sum_{i=1}^{N} y_{i}, w \rightarrow \infty
\end{array}\right.
\end{aligned}
$$

Здесь $w$ - ширина окна сглаживания, которая найдена из минимума относительного значения ошибки относительно выбранного $w ; y_{j}(j=1$, $2, \ldots, N)$ - начальная («зашумленная») функция, содержащая ВЧ флуктуации; $Y_{j}$ - соответствует сглаженной функции. Интересно отметить, что при $w>1$ сглаженная функция совпадает со средним арифметическим $y_{j}$, а при $w$ стремящемуся к нулю сглаженная функция совпадает с исходной, т. е. $Y_{j}=y_{j}$ для всего числа доступных точек $j$. Численные расчеты показывают, что если требуется, чтобы коэффициент корреляции Пирсона между начальной и сглаженной функциями приблизительно равнялся 0.98, то для представленных данных оптимальное значение близко к округленному значению $w \approx 0.5$. 


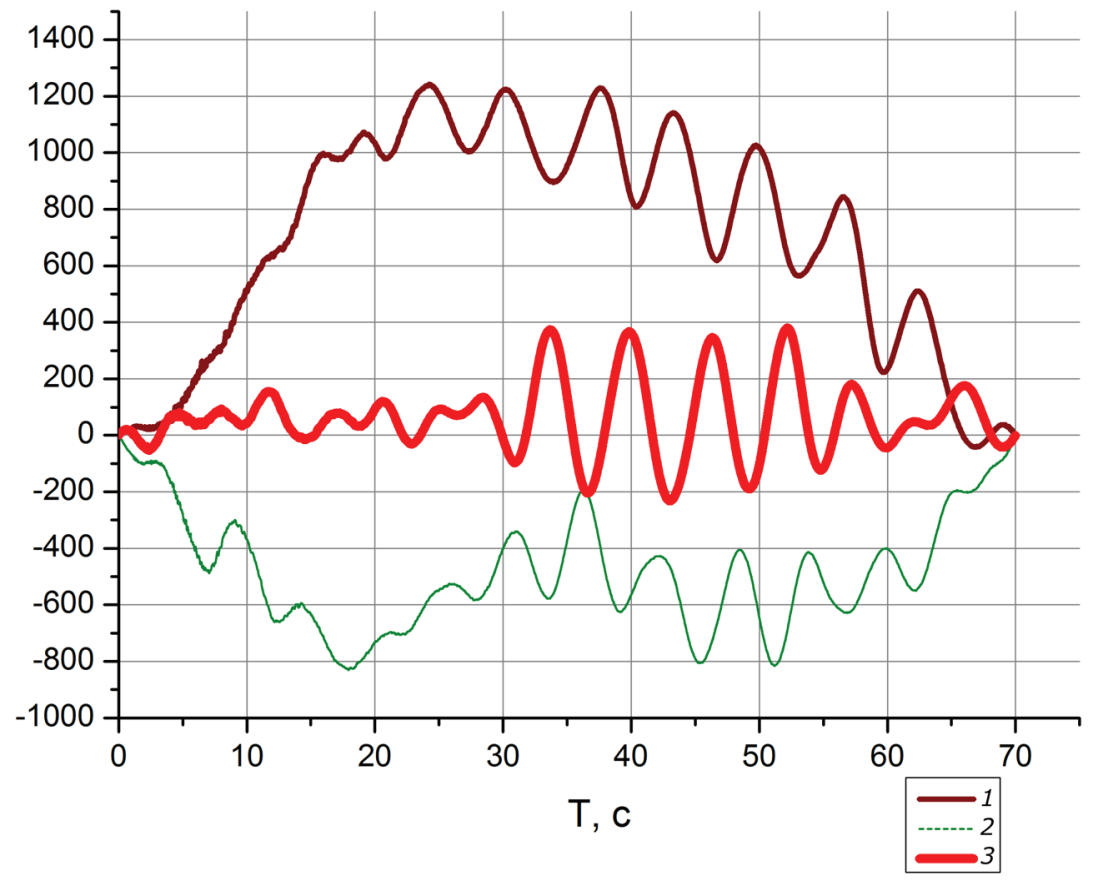

Рис. 2. Процедура интегрирования записи землетрясения по трем компонентам: (линии 1 - ННЕ, $2-\mathrm{HHN}, 3-\mathrm{HHZ})$. Заметно, что после интегрирования высокочастотные (ВЧ) биения исчезают, и количественное описание этих интегрированных волн может быть также реализовано с помощью подхода НАЧАСС (неортогонального амплитудно-частотного анализа сглаженных сигналов).

Fig. 2. The integration for the earthquake recording from three components: (lines $1-\mathrm{HHE}, 2-$ HHN, $3-\mathrm{HHZ}$ ). Notice that after integration high-frequency (HF) beats disappear, and a quantitative description of these integrated waves can also be implemented using the approach NAFASS (nonorthogonal amplitude-frequency analysis of the smoothed signals).
После сглаживания сигнала его можно представить в виде отрезка ряда, представляющего собой мульти-периодическое разложение и используемое в дальнейшем в качестве подгоночной функции. Это разложение осуществляется с помощью НАЧАСС алгоритма, описанного в работах (Nigmatullin, 2014, 2018). Суть предлагаемого подхода заключается в следующем. Предлагаемый алгоритм помогает найти разложение для многочастотных сигналов, которые изначально не могут быть приближенно описаны конечным набором членов обычного ряда Фурье. Как известно, разложение Фурье основано на априорном предположении о том, что данный сигнал является периодическим с периодом равным временному интервалу, занимаемому рассматриваемым сигналом. В большинстве случаев это предположение является необоснованным и поэтому разложение Фурье в качестве подгоночной функции не используется; оно применяется большинством исследователей в качестве независимого источника информации для анализа сложных сигналов, при этом часть вычисляемого Ф-спектра может содержать частоты, которые не содержатся в спектре анализируемого сложного сигнала.
НАЧАСС разложение представляет собой подгоночную функцию, причем мультичастотный спектр $\Omega_{k}$ не предполагается, a приближенно вычисляется из итерационной формулы (15) работы (Nigmatullin, 2018). Следует особо подчеркнуть, что функциональная зависимость $\Omega_{k}$ от волнового вектора $k$ находится из принципа, что сложный сигнал представляет собой совокупность всевозможных обобщенных биений, т.е. описывается произведением обобщенных биений. Математически, этот принцип может быть выражен в виде:

$$
\begin{gathered}
\prod_{i=1}^{p} \Delta_{\alpha_{i}}\left(\Omega_{k}\right)=0, \\
\Delta_{\alpha_{i}}\left(\Omega_{k}\right) \equiv(E-\alpha) \Omega_{k}=\Omega_{k+1}-\alpha \Omega_{k} .
\end{gathered}
$$

Здесь параметр $\alpha$ может принимать произвольные значения, включая даже пару комплексно-сопряженных величин, $E-$ определяет оператор сдвига.

НАЧАСС разложение позволяет представить широкий класс случайных сигналов, имеющих мультичастотный спектр в виде следующего разложения:

$$
S(t) \cong F\left(t ; \Omega_{k}, K\right)=A_{0}+\sum_{k=0}^{K-1}\left[A c_{k} \cos \left(\Omega_{k} t\right)+A s_{k} \sin \left(\Omega_{k} t\right)\right],
$$

где $A_{0}, A c_{k}, A s_{k}(k=0,1, \ldots, K$-1) - искомые амплитуды разложения заданного отрезка ряда (3), $S(t)$ - исходный сигнал, $\Omega_{k}(k=0,1, \ldots, K-1)-$ закон дисперсии, который вычисляется по алгоритмической формуле (15) приведенной в работе
(Nigmatullin, 2018). Предельную моду $K$ можно найти из минимизации средней относительной ошибки, если учесть доверительный интервал локализации вычисленного спектра $\Omega_{k}$ и взять число мод $K$, обеспечивающих приемлемую 
точность подгонки. Конечная мода $K$, входящая в разложение (3), вычисляется из выражения для средней относительной ошибки:

$$
\operatorname{Re} l \operatorname{Err}(\%)=\left(\frac{\operatorname{stdev}(S(t)-F(t ; K))}{\operatorname{mean}|S(t)|}\right) \cdot 100 \%,
$$

что минимизирует значение относительной погрешности (выраженное в процентах) относительно значения конечной моды $K$. Для вычислительных целей достаточно сохранять ошибку, определенную в выражении (4), в пределах (1-10\%), чтобы значение $K$ было меньше $N_{r} / 10$ (где $N_{r}$ число «жирных» (укрупненных) точек). Чтобы уменьшить число мод, можно предварительно сгладить сигнал (в данном случае огибающую), и найти его АЧХ с помощью НАЧАСС разложения (рис. 3). После вычисления спектра частот $\Omega_{k}$, искомые амплитуды, фигурирующие в выражении (2), вычисляются с помощью обычного линейного метода наименьших квадратов (ЛМНК). В рамках подхода НАЧАСС существует возможность найти подходящий искомый спектр частот $\Omega_{k}$, если принять следующий принцип: в сложной системе могут быть реализованы всевозможные биения, определяемые математически с помощью формулы (2). С использованием этого принципа можно показать, что для заданного конкретного случая набор частот получается из следующего разностного уравнения:

$$
(E-1)^{3} \Omega_{k}=0
$$

или

$$
\Omega_{k+3}=3 \Omega_{k+2}-3 \Omega_{k+1}+\Omega_{k} .
$$

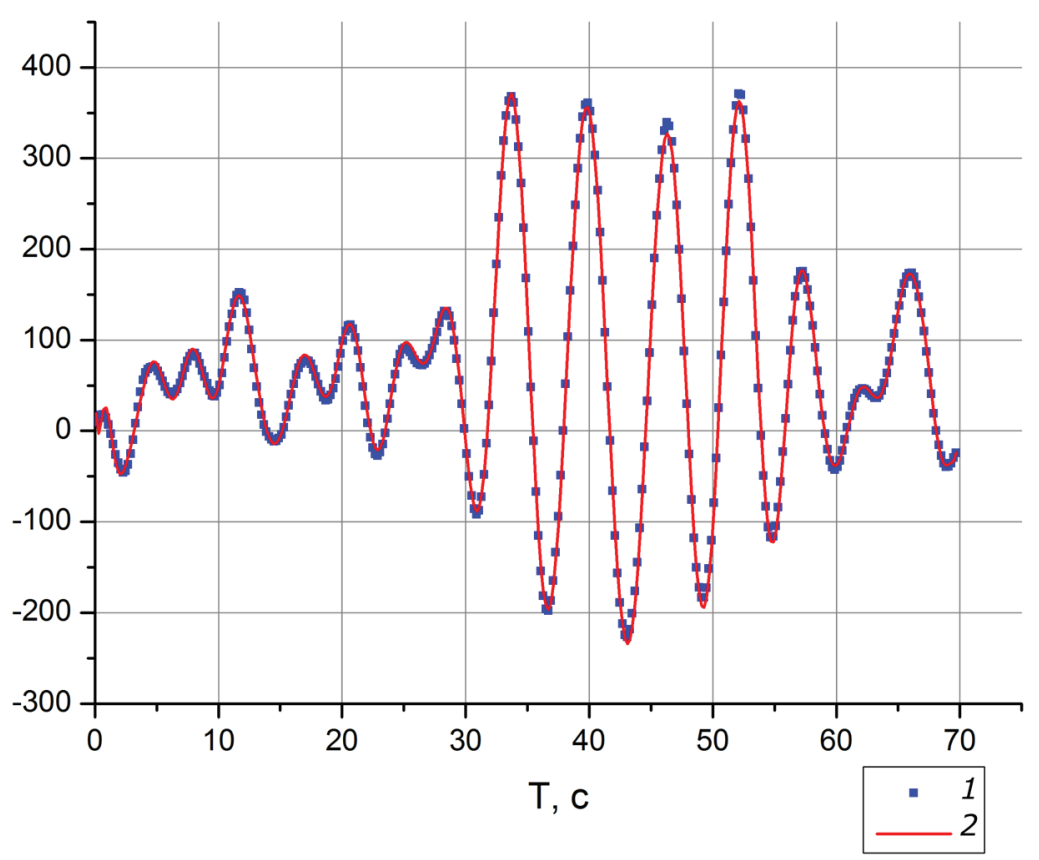

Здесь $E$ оператор сдвига, который определен в (2). Решения линейных разностных уравнений такого типа широко известны и описаны в многочисленных справочниках, например, (Корн, Корн, 1973).

Теперь обратимся к форме закона дисперсии частот $\Omega_{k}$. Поскольку, как уже было сказано, закон дисперсии $\Omega_{k}$ связан с волновым вектором $k$ функциональной зависимостью, вытекающей из принципа (2), то для его нахождения, необходимо воспользоваться общим методом решения разностных уравнений, описанным в справочнике (Корн, Корн, 1973). Разностное уравнение (5) имеет 3-х кратно-вырожденный корень $\lambda=1$. Поэтому решение, определяющее зависимость $\Omega_{k}$ при $p=3$, имеет вид (6). Если корень 4-Х кратно вырожден, то решение описывается полиномом 3-го порядка и приведено во второй строке (6). Численные расчеты показывают, что увеличение порядка полинома не приводит к существенному увеличению точности, поэтому для вычислительных целей достаточно ограничиться решением, представленным первой строкой выражения (6).

$$
\begin{aligned}
& \Omega_{k}=a_{0}+a_{1} k+a_{2} k^{2}, \text { для } p=3, \\
& \Omega_{k}=a_{0}+a_{1} k+a_{2} k^{2}+a_{3} k^{3}, \text { для } p=4 .
\end{aligned}
$$

Неизвестные параметры $\left(a_{0,1,2}\right)$, входящие в (6) могут быть найдены из системы линейных уравнений (7),

$$
\begin{aligned}
& a_{0}=\Omega_{0}, \\
& a_{0}+a_{1}+a_{2}=\Omega_{1}, \\
& a_{0}+2 a_{1}+4 a_{2}=\Omega_{2},
\end{aligned}
$$

если изначальные частоты $\Omega_{0,1,2}$ известны точно. В случае их приближенного вычисления они оцениваются при помощи ЛМНК. Поэтому,

Рис. 3. Подгонка (точечная линия 1) для сигнала от землетрясения (сплошная линия 2), записанного на вертикальной компоненте (HHZ). Чтобы получить значение относительной ошибки RelErr $<5 \%$ (по выражению (3)), требуется предельное значение моды $\mathrm{K}=26$.

Fig. 3. Fitting function curve (dotted line 1) for an earthquake signal (solid line 2) recorded at the vertical component (HHZ). To obtain the relative error value RelErr $<5 \%$ (from equation (3)), the limit value of the mode $K=26$ is required. 


\section{АНАЛИТИЧЕСКОЕ ОПИСАНИЕ}

используя подход НАЧАСС, можно найти АЧХ (зависимость $A c_{k}, A s_{k}, A m d_{k}=\sqrt{A c_{k}^{2}+A s_{k}^{2}}$ ) и искомый закон дисперсии $\Omega_{k}$ от целого числа текущей моды $k$ для верхней НЧ огибающей (рис. 4).

В полной аналогии с этими расчетами можно оценить НЧ составляющую для нижней огибающей $E d n$.

Шаг 3. Оценка высокочастотной составляющей. Для оценки ВЧ составляющей используется следующее выражение, основанное на идее нормировки:

$$
\begin{aligned}
& E_{r}(t) \cong \operatorname{Emn}_{r}(t)+H F_{r}(t)\left(E m n_{r}(t)-E d n_{r}\right), \\
& E_{n}(t)=\frac{1}{2}\left(\operatorname{Eup}_{r}(t)+E d n_{r}(t)\right), H F_{r}(t) \in[-1,1] .
\end{aligned}
$$

Выражение (8) описывает приведенный сигнал $E_{r}(t)$ начальной формы записи землетрясения. В качестве ВЧ составляющей можно взять набор точек средних значений $b_{\text {mean }}=\operatorname{mean}\left(b_{1}, b_{2}\right.$, $\ldots, b_{M}$ ), образованный приведенными точками в каждом сегменте $r=0,1, \ldots, R-1$, а затем нормировать эту кривую «средних значений» на интер- вале $(-1,1)$. Следует заметить, что в общем случае нормированная кривая $\operatorname{Enrm}_{r}(t)$ не совпадает с $E_{n r m}(t)$, которое определяется выражением (8). Подсчитав количество нулей для данного $\operatorname{Enrm}_{r}(t)$ и установив пределы $(-L, L)$ близкими к нулевому значению, можно перейти к идентификации всех малых значений, расположенных в этом интервале. Для тех же значений, что содержат «истинные» корни, выбирается неизвестное значение $L$. Его можно выбрать из критерия, согласно которому погрешность вычисленных корней функции Emean $_{r}(t)$ не должна превышать значение $2,5 \%$ от его максимального значения. Исходя из заданного порога, положим $L=0,025$. Внимательный анализ позволяет заключить, что распределение корней генерируется приблизительно набором отрезков $(s=0,1,2, \ldots, S)$ $R t_{s}=A_{s} k_{s}+B_{s}$, где значение

$$
k_{s} \in\left[k_{\min }^{(s)}, k_{\max }^{(s)}\right] .
$$

С другой стороны, аналогичная структура корней отвечает выражению (9) и записывается в виде произведения:

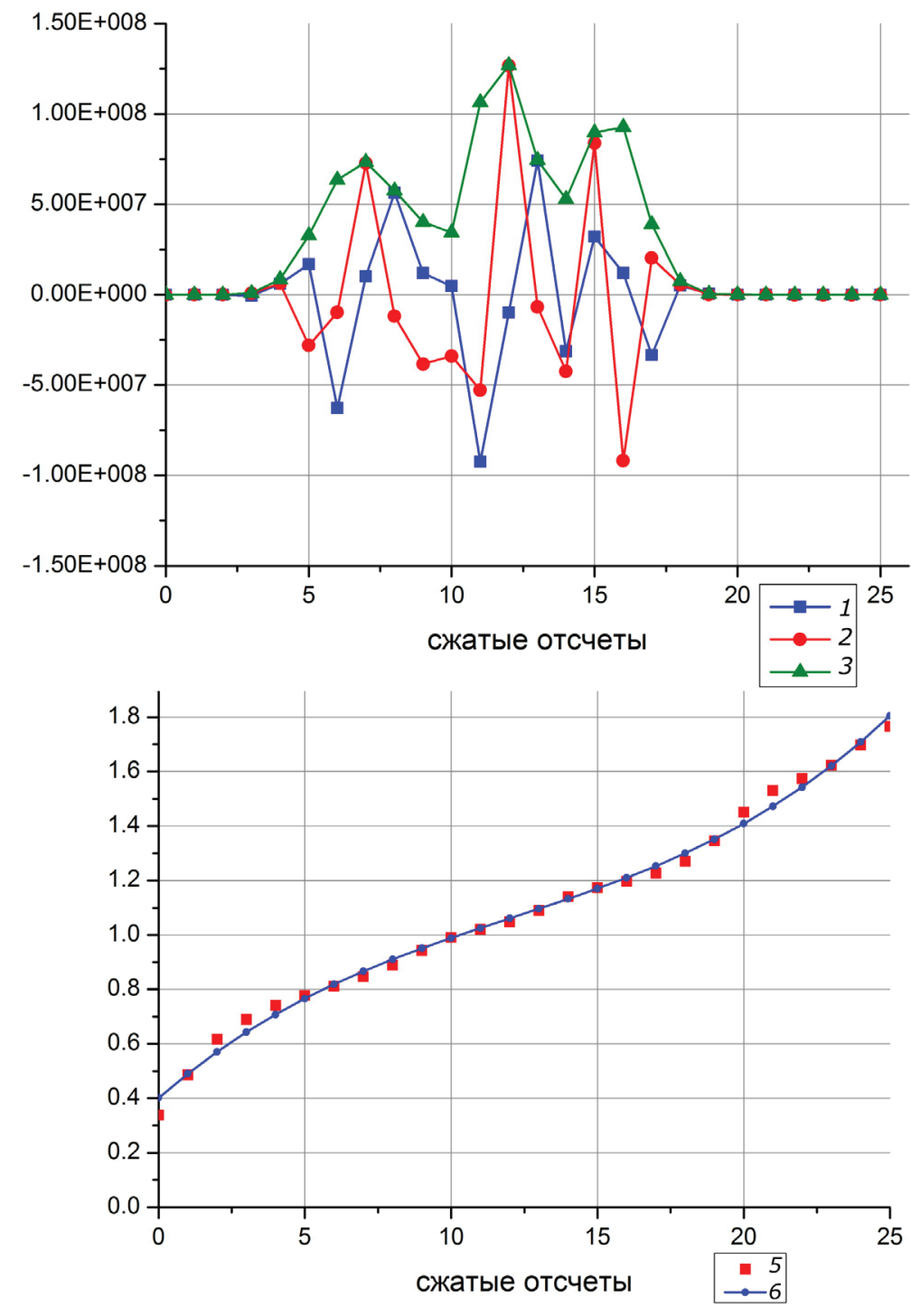

Рис. 4. Амплитудно-частотная характеристика (АЧХ) для верхней огибающей (a) сигнала, изображенного на рис. 2 , и закон дисперсии ( $\Omega$ показана линией 6$)$ и его подгонка $\left(\Omega_{f t}\right.$ показана линией 5$)$ для записи сигнала от землетрясения на вертикальной компоненте HHZ (б) (конечная мода $K=34$ ). Функция подгонки для законов дисперсии на рис. $4 a$ описывается многочленом третьего порядка: 1 - амплитуды в разложении при косинусе, $A c_{s}, 2$ - амплитуды в разложении при синусе $A s_{s}, 3$ - квадратный корень из первых двух амплитуд:

$$
A m d_{S}=\sqrt{A c_{S}^{2}+A s_{S}^{2}} .
$$

Fig. 4. Amplitude-frequency characteristic (AFC) for the upper envelope (a) of the signal shown in Fig. 2, and the dispersion law $(\Omega$ is shown as line 6 ) and its fitting ( $\Omega \mathrm{ft}$ is shown as line 5) for recording of the signal from the earthquake on the vertical component HHZ (б) (terminal mode $K=34$ ). The fitting function for the dispersion laws in Fig. $4 a$ is described by a thirdorder polynomial: 1 - amplitudes in the expansion with cosine, $A c_{s}, 2$ - amplitudes in the decomposition with sine $A s_{s}$, 3 - square root of the first two amplitudes $A m d_{S}=\sqrt{A c_{S}^{2}+A s_{S}^{2}}$. 


$$
H F_{r}(t) \cong \Psi_{r}(t)=\prod_{s=0}^{S} \cos \left(\omega_{s} t_{r}-\Phi_{s}\right),-1 \leq \Psi_{r}(t) \leq 1,
$$

где $S$ - предельное значение переменной $s$, при которой данное распределение корней заканчивается. Функция $\Psi_{r}(t)$, которая имеет следующий вид распределения корней:

$$
\begin{aligned}
& \omega_{s} R t_{s}-\Phi_{s}=\frac{\pi}{2}+k_{s} \pi, k_{s}=0, \pm 1, \ldots \\
& R t_{s}=\frac{\pi}{\omega_{s}} k_{s}+\frac{1}{\omega_{s}}\left(\frac{\pi}{2}+\Phi_{s}\right) .
\end{aligned}
$$

Объединяя эти выражения, вычисляя неизвестные наклоны $A_{s}$ и отсечки $B_{s}$ для данного $S$ (рассчитывается приблизительно из замены данных корней распределения $R t_{s}$ функции $\operatorname{Enrm}_{r}(t)$ набором кусочно-линейных сегментов $\left.R t_{s}=A_{s} k_{s}+B_{s}\right)$, можно приближенно оценить искомые частоты $\omega_{s}$ и фазы $\Phi_{S}$ из соотношений:

$$
\omega_{s}=\frac{\pi}{A_{s}}, \Phi_{s}=\omega_{s} B_{s}-\frac{\pi}{2} .
$$

Произведенные расчеты по предложенному выше алгоритму показывают, что их спектры не являются простыми и не могут быть представлены обычным преобразованием Фурье. Применение Фурье-разложения не позволяет подогнать верхнюю и нижнюю огибающую УПС. Его формальное применение создает избыточное количество частот, которое может быть и не связано с истинным частотным спектром, формирующим зарегистрированную волну от землетрясения. Верхняя и нижняя огибающие волны землетрясения могут быть подогнаны с помощью многочастотного разложения (3). В свою очередь, ВЧ составляющую удобно представить приближенно с помощью произведения (9). Его получают восстановлением из анализа распределения корней, принадлежащего нормированной средней составляющей $E_{r}(t)$, которая вычисляется из выражения (8).

\section{ВЫВОДЫ}

Как следует из проведенного анализа, предлагаемый подход с использованием метода НАЧАСС позволяет количественно описать сложную структуру волн от землетрясений и найти их АЧХ. Сравнение с исходными сигналами землетрясений показывает, что количественный анализ этих интегрированных размерностей проще, и эта особенность может быть использована и для целей классификации сигналов.

Полученная подгоночная функция является сжатой в 30 раз копией исходного землетрясения (рис. 3). Таким образом, особенности сигналов, генерируемых землетрясениями и относящихся к сложному классу биений (принадлежащему некоторому классу УПС), можно воспроизвести с относительно высокой точностью, с ошибкой, лежащей в интервале (1-10\%). Такой способ описания сигналов землетрясений с помощью НАЧАСС алгоритма открывает новые возможности в геофизических исследованиях сейсмоактивных регионов, в том числе, осуществляемых Научной станцией РАН на территории ТяньШаня.

Проведенные исследования выполнены в рамках государственного задания Научной станции РАН «Изучение геодинамических, сейсмических и геофизических процессов как основы прогноза землетрясений (включая моделирование неупругих процессов в сейсмогенерирующих зонах)» (Тема IX.136. 0155-2019-0003).

\section{Список литературы [References]}

Асминг В.Э. Создание программного комплекса для автоматизации детектирования, локации и интерпретации сейсмических событий и его использование для изучения сейсмичности северо-западного региона / Дис. канд. физ.-мат. наук. Москва, 2004. 137 с. [Asming V.E. Creating a software system for automating the detection, location and interpretation of seismic events and its use to study the seismicity of the north-western region / Dis. kand. fiz.-mat. nauk. Moscow, 2004. 137 p. (in Russian)].

Баранов C.B. Построение систем автоматического детектирования и локации сейсмических событий в реальном времени. Автореф. дис. канд. физ.-мат. наук. Апатиты, 2002. 36 с. [Baranov S.V. Postroyeniye sistem avtomaticheskogo detektirovaniya i lokatsii seysmicheskikh sobytiy v real'nom vremeni. Avtoref. dis. kand. fiz.-mat. nauk. Apatity, 2002. 36 p. (in Russian)].

Березина А.В., Брагин В.Д., Першина Е.В., Рыбин А.К. Сейсмическая база данных по материалам сетей KNET и KRNET // Вестник Кыргызско-Российского славянского университета. 2013. Т. 13. № 7. C. 84-90 [Berezina A.V., Bragin V.D., Pershina E.V., Rybin A.K. Seismic database on the basis of materials of seismic networks KNET and KRNET // Vestnik Kyrgyzsko-Rossiyskogo slavyanskogo universiteta. 2013. V. 13. № 7. P. 84-90 (in Russian)].

Зосимов В.В., Лямшев Л.М. Фракталы в волновых процессах // Успехи физических наук. 1995. T. 165. № 4. C. 361-402. https://doi.org/10.3367/ UFNr.0165.199504a.0361 [Zosimov V.V., Lyamshev L.M. Fractals in wave processes // Physics-Uspekhi. 1995. V. 38. № 4. P. 347-384. https://doi.org/10.1070/ PU1995v038n04ABEH000080].

Конечная Я.В. Особенности природной сейсмичности западного сектора Арктической зоны РФ по данным станций Баренц-региона / Автореф. дис. канд. техн. наук. Архангельск, 2015. 24 с. [Konechnaya Ya.V. Osobennosti prirodnoy seysmichnosti zapadnogo 


\section{АНАЛИТИЧЕСКОЕ ОПИСАНИЕ}

sektora Arkticheskoy zony RF po dannym stantsiy Barents-regiona / Avtoref. dis. kand. tekhn. nauk. Arkhangelsk, 2015. 24 p. (in Russian)].

Корн Г., Корн Т. Справочник по математике для научных работников и инженеров. М.: Наука, 1973. 832 c. [Korn G.A., Korn T.M. Mathematical handbook: For scientists and engineers, 1968. McGraw-Hill Book Company, New York, USA. 1130 p.].

Короновский А.А., Храмов А.E. Непрерывный вейвлетный анализ и его приложения. М.: Физматлит, 2003. 176 с. [Koronovskiy A.A., Khramov A.E. Nepreryvnyy veyvletnyy analiz i yego prilozheniya. Moscow: Fizmatlit, 2003. 176 p. (in Russian)].

Левин Б.В., Сасорова Е.В., Борисов С.А., Борисов А.С. Оценка параметров слабых землетрясений и их сигналов // Вулканология и сейсмология. 2010. № 3. C. 60-70 [Levin B.V., Borisov S.A., Borisov A.S., Sasorova E.V. Estimating the parameters of small earthquakes and their signals // Journal of Volcanology and Seismology. 2010. V. 4. № 3. P. 203-212. https:// doi.org/10.1134/S074204631003005X].

Любушин A.A. Анализ данных систем геофизического и экологического мониторинга. М.: Наука, 2007. 228 c. [Lyubushin A.A. Analiz dannykh sistem geofizicheskogo i ekologicheskogo monitoringa. Moscow: Nauka, 2007. 228 p. (in Russian)].

Малла C. Вейвлеты в обработке сигналов. М.: Мир, 2005. 671 c. [Malla $S$. A Wavelet Tour of Signal Processing. Academic Press, $2^{\text {nd }}$ edition. 1999. 832 p.].

Морозов A.H. Метод идентификации взрывной сейсмичности на территории Архангельской области // Вестник Камчатской региональной организации «Учебно-научный центр». Серия: Науки о Земле. 2008. № 1. Вып. 11. С. 177-184 [Morozov A.N. Method of identification of explosive seismicity on territories of the Arkhangelsk region // Vestnik KRAUNTs. Nauki o Zemle. 2008. № 1(11). P. 177-184 (in Russian)].

Морозов Ю.В., Спектор A.A. Классификация объектов на основе анализа спектральных характеристик огибающих сейсмических сигналов // Автометрия. 2017. Т. 53. № 6. С. 49-56. https://doi.org/10.15372/AUT20170606 [Morozov Y.V., Spektor A.A. Object Classification Based on Analysis of Spectral Characteristics of Seismic Signal Envelopes // Optoelectronics, Instrumentation and Data Processing. 2017. V. 53. № 6. P. 576-582. https:// doi.org/10.3103/S8756699017060061].

Мухамадеева В.А., Сычева Н.А. О предварительных результатах анализа каталогов землетрясений для территории Бишкекского геодинамического полигона // Вестник Кыргызско-Российского славянского университета. 2017. Т. 17. № 8. С. 170-177 [Mukhamadeeva V.A., Sycheva N.A. Preliminary results of analysis of the earthquakes catalogs for the territory of Bishkek Geodynamic Proving Ground // Vestnik Kyrgyzsko-Rossiyskogo slavyanskogo universiteta. 2017. V. 17. № 8. P. 170-177 (in Russian)].
Новиков И.Я., Протасов В.Ю., Скопина М.А. Теория всплесков. М: Физматлит, 2005. 616 с. [Novikov I.Ya., Protasov V.Yu., Skopina M.A. Teoriya vspleskov. Moscow: Fizmatlit, 2005. 616 p. (in Russian)].

Родионов E.A. О применении вейвлетов к цифровой обработке сигналов // Известия Саратовского университета. Новая серия. Серия «Математика. Механика. Информатика». 2016. Т. 16. Вып. 2. С. 217-225. https://doi.org/10.18500/1816-9791-2016-162-217-225 [Rodionov E.A. On Applications of wavelets in Digital Signal Processing // Izvestiya Saratovskogo Universiteta Novaya Seriya - Matematika Mekhanika Informatika. 2016. V. 16. № 2. P. 217-225. https://doi. org/10.18500/1816-9791-2016-16-2-217-225 (in Russian)].

Рыбин А.К., Баталева Е.А., Леонов М.Г. и др. Современная геодинамическая активность земной коры северного Тянь-Шаня и опасные геологические процессы // Вестник Кыргызско-Российского славянского университета. 2016. Т. 16. № 5. C. 157-163 [Rybin A.K., Bataleva E.A., Leonov M.G. et al. Modern geodynamic activity of the Northern Tien Shan earth crust and dangerous geological processes // Vestnik Kyrgyzsko-Rossiyskogo slavyanskogo universiteta. 2016. V. 16. № 5. P. 157-163 (in Russian)].

Федер Е. Фракталы. М.: Мир, 1991. 254 с. [Feder J. Fractals. Springer US. 1988. 284 p. https://doi. org/10.1007/978-1-4899-2124-6].

Фролова А.Г, Джанузаков К.Д., Першина Е.В. Сейсмичность территории Кыргызстана // Известия Национальной Академии Наук Кыргызской Республики. 2012. № 3. С. 45-49 [Frolova A.G, Dzhanuzakov K.D., Pershina E.V. Seysmichnost' territorii Kyrgyzstana // Izvestiya Natsional'noy Akademii Nauk Kyrgyzskoy Respubliki. 2012. № 3. P. 45-49 (in Russian)].

Abdrakhmatov K., Havenith H.-B., Delvaux D., Jongmans D., Trefois $P$. Probabilistic PGA and arias intensity maps of Kyrgyzstan (Central Asia) // Journal of Seismology. 2003. V. 7. Iss. 2. P. 203-220. https://doi. org/10.1023/A:1023559932255.

Geller R.J., Mueller C.S. Four similar earthquakes in central California // Geophysical Research Letters. 1980. V. 7. Iss. 10. P. 821-824. https://doi.org/10.1029/ GL007i010p00821.

Nigmatullin R.R., Rakhmatullin R. Detection of quasiperiodic processes in repeated-measurements: new approach for the fitting and clusterization of different data // Communications of Nonlinear Science and Numerical Simulation. 2014. V. 19. Iss. 12. P. 4080-4093. https://doi.org/10.1016/j.cnsns.2014.04.013.

Nigmatullin R.R., Toboev V.A., Lino P., Maione G. Reduced fractal model for quantitative analysis of averaged micromotions in mesoscale: Characterization of blow-like signals // Chaos, Solitons \& Fractals. 2015. V. 76. Iss. 7. P. 166-181. https://doi.org/10.1016/j. chaos.2015.03.022.

Nigmatullin R.R., Gubaidullin I.A. NAFASS: Fluctuation spectroscopy and the Prony spectrum for description 


\title{
РЫБИН и др.
}

of multi-frequency signals in complex systems // Communications in Nonlinear Science and Numerical Simulation. 2018. V. 56. Iss. 3. P. 252-269. https://doi. org/10.1016/j.cnsns.2017.08.009.

Schaff D.P., Beroza G.C. Coseismic and postseismic velocity changes measured by repeating earthquakes // Journal
Geophysical Research. 2004. V. 109. Iss. B10. B10302. https://doi:10.1029/2004JB003011.

Yoon C., O'Reilly O., Bergen K., Beroza G. Earthquake detection through computationally efficient similarity search // Science Advances. 2015. V. 1. Iss. 11. e1501057. https://doi.org/10.1126/sciadv.1501057.

\section{ANALYTICAL DESCRIPTION OF THE SEISMIC SIGNALS BASED ON THE NONORTHOGONAL AMPLITUDE-FREQUENCY ANALYSIS OF THE SMOOTHED SIGNALS}

\author{
A.K. Rybin ${ }^{1}$, R.R. Nigmatullin ${ }^{2}$, K.S. Nepeina ${ }^{1}$, \\ P.A. Kaznacheev ${ }^{3}$, P.N. Alexandrov ${ }^{4}$ \\ ${ }^{1}$ Research Station of the Russian Academy of Sciences, Bishkek; e-mail: rybin@gdirc.ru \\ ${ }^{2}$ Kazan National Research Technical University named after A.N. Tupolev - KAI, Kazan \\ ${ }^{3}$ Schmidt Institute of Physics of the Earth of the Russian Academy of Sciences, Moscow \\ ${ }^{4}$ Geoelectromagnetic Research Centre of the Russian Academy of Sciences, Moscow, Troitsk
}

\begin{abstract}
The paper presents a new approach to the description of earthquake seismic signals based on the nonorthogonal amplitude-frequency analysis of the smoothed signals (NAFASS) method. The authors consider an example of the processing of one of earthquake's records that occurred in the Tien Shan territory. The earthquake signals were recorded at the Karagai-Bulak seismic station, which is a part of the digital telemetry network KNET, located in the Northern Tien Shan area. The paper presents the results from the first stage of creation of a collection of blow-like signals with beatings (such as earthquakes) «fingerprints», which will provide additional information characterizing the modern geodynamic processes of the Tien Shan. Such analysis of seismic signals for the Tien Shan earthquakes has not been carried out yet. The authors hope that using the reduced fractal model on the base of NAFASS method will help avoid extensive calculations of standard methods and directly construct a quantitative description of earthquakes in the terms of a common set of fitting parameters by creating a «waveform portrait» of a seismic event.
\end{abstract}

Keywords: blow-like signals, NAFASS method, earthquake, Tien Shan. 